\title{
Band bending in the initial stages of Schottky-barrier formation for gallium on Si(113)
}

\author{
P. Althainz, U. Myler, and K. Jacobi \\ Fritz-Haber-Institut der Max-Planck-Gesellschaft, Faradayweg 4-6, D-1000 Berlin 33, West Germany
}

(Received 12 May 1989; revised manuscript received 3 August 1989)

\begin{abstract}
We present angle-resolved ultraviolet and soft-x-ray photoelectron spectroscopy results for the Schottky-barrier formation of $\mathrm{Ga}$ on $p$-type $\mathrm{Si}(113)$. For the first 0.08 monolayer of $\mathrm{Ga}$, the band bending increases. For higher coverages, it decreases monotonically until it reaches its final value at about 2 monolayers. This change of band bending is found for a Si surface for the first time and supports a recent model calculation. The final barrier height is $0.32 \pm 0.10 \mathrm{eV}$, in good agreement with the values found for low-index surfaces.
\end{abstract}

\section{INTRODUCTION}

Semiconductor interfaces are of great technical importance. Therefore, much effort has been invested in studying such interfaces on a microscopic scale during the last few years. ${ }^{1}$ Here, we concentrate on the metalsemiconductor interface or Schottky contact. The flow of current across such a contact is determined by the Schottky-barrier (SB) height which is the energy difference between the Fermi level and the conduction (valence) band in the case of an $n$-type ( $p$-type) semiconductor. The aluminum, gallium, or indium $s p$-metal adlayers on silicon are well-known examples of almost ideal and abrupt SB's. ${ }^{2-4}$ Since 1964 when Lander and Morrison ${ }^{5}$ addressed the problem of trivalent metals on the $\mathrm{Si}(111)$ surface, such topics as SB formation, ${ }^{6-12}$ growth modes, ${ }^{13,14}$ geometric structure at submonolayer coverages, $^{15-19}$ and surface electronic structure ${ }^{3,4,20-26}$ have been studied for group-III metals on $\mathrm{Si}$, using a variety of surface-sensitive tools. The chemisorption of trivalent metals on silicon was found to take place in two steps. The first atoms at submonolayer coverages form bonds to the substrate with more covalent than metallic character. In the second step, i.e., at a coverage higher than $\frac{1}{3}$ monolayer (ML), the bonding character changes. At higher coverages a metallic adlayer and metal-induced in-

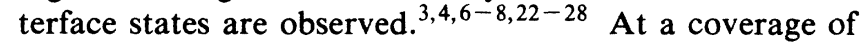
$\frac{1}{3} \mathrm{ML}, \mathrm{Al}, \mathrm{Ga}$, and In form ordered overlayers on $\mathrm{Si}(111)$. Recently, the atomic arrangement of submonolayer coverages of these metals on $\mathrm{Si}(111)$ surfaces has been investigated with low-energy electron diffraction (LEED), ${ }^{15}$ reflection high-energy electron diffraction (RHEED), ${ }^{16}$ the $\mathrm{X}$-ray standing-wave technique (XSW), ${ }^{17,18}$ and scanning tunneling microscopy (STM). ${ }^{19}$ In the course of a project on higher-index $\mathrm{Si}$ and $\mathrm{GaAs}$ surfaces we are studying the electronic and structural properties of the $\mathrm{Si}(113)$ surface. $^{29}$ The $\mathrm{Si}(113)$ surface is interesting since the unit cell of the ideal bulk truncated surface contains one threefold- and one twofold-coordinated atom, making it a candidate for heteroepitaxy of III-V compounds. The real surface is reconstructed into a $(3 \times 2)$ superstructure after annealing to temperatures higher than $1050 \mathrm{~K}$. The $(3 \times 2)$ surface is characterized by a strong surface resonance peaked at $0.9 \mathrm{eV}$ below $E_{F}($ at $300 \mathrm{~K})$ which is assigned to the Si dangling bonds. ${ }^{29}$

Here we present data for the $\mathrm{Ga} / \mathrm{Si}$ (113) SB formation. Various coverages of $\mathrm{Ga}$ on silicon were prepared, ranging from about a tenth to several ML. It turned out that the band bending observed at very low coverages is quite different from results of other authors, whereas the value of the final barrier height is in good agreement with reported values for $\mathrm{Si}(111)$ surfaces. Recently, Klepeis and Harrison calculated the electronic structure for such a SB between column-III metals and $\mathrm{Si}^{30}$ We will show that this model is strongly supported by our measurements. Also, several recent results for SB's on GaAs at lower temperatures ${ }^{31-34}$ are similar to our findings and have been interpreted along similar lines. ${ }^{33-35}$

\section{EXPERIMENT}

The measurements were performed in an UHV $\mu$-metal chamber with a base pressure of $5 \times 10^{-11}$ Torr. The surface-analytical equipment consisted of an electron spectrometer for Auger-electron spectroscopy (AES) and angle-resolved ultraviolet photoelectron spectroscopy (ARUPS) as well as optics for low-energy electron diffraction. The energy resolution of the hemispherical electron-energy analyzer (ADES 400, VG Scientific) was chosen to be $100 \mathrm{meV}$ during the ARUPS measurements and $2 \mathrm{eV}$ during AES. Synchrotron radiation was used for ARUPS from the TGM 3 (toroidal grating monochromator) beamline at the Berlin electron storage ring BESSY (Berliner Elektronenspeicherring-Gesellschaft für Synchrotronstrahlung). The energy-dependent resolution of the monochromator contributed to the overall energy resolution, which was $100 \mathrm{meV}$ at $\hbar \omega=11.7 \mathrm{eV}, 300 \mathrm{meV}$ at $30 \mathrm{eV}$, and $400 \mathrm{meV}$ at $108 \mathrm{eV}$. The energy of the incident light was calibrated by measuring the Fermi level energy of the tantalum sample holder with the first and second diffraction order of the monochromator grating. The monochromator had three built-in gratings covering different energy ranges. The high-energy grating, used for measurements with $108 \mathrm{eV}$ photon energy, could not be calibrated in this way due to a lack of intensity in the second order. Core-level UP spectra taken with this grating yield only relative binding-energy shifts. All ARUP and soft-x-ray photoemission (SXP) spectra were record- 
ed in normal emission.

The preparation of the $p$-doped $\left[2.5 \times 10^{17}\right.$ (atoms boron) $/ \mathrm{cm}^{3}$ ] $\mathrm{Si}(113)$ sample is described elsewhere. ${ }^{29}$ Briefly, the clean Si(113) surface was obtained by argon-ion sputtering and annealing up to $1200 \mathrm{~K}$. A high surface state intensity in ARUPS and a $(3 \times 2)$ LEED pattern indicated a clean sample. Gallium (99.9999\% pure) was vapor deposited from an effusion cell heated by electron bombardment. The temperature of the cell was held constant by regulating the power input, thus maintaining a uniform deposition rate. All evaporations were made with the substrate kept at room temperature. During the evaporation of gallium the pressure did not exceed $2 \times 10^{-10}$ Torr. The cleanliness of the evaporated gallium was checked by UPS. Spectra of thick gallium layers showed no signs of contamination, although gallium UP spectra are sensitive to oxygen contamination within coverages of $0.01 \mathrm{ML}$.

\section{RESULTS}

Auger-electron spectra were taken to determine the thickness of the $\mathrm{Ga}$ overlayer on the silicon substrate. Figure 1 exhibits the curves of the decreasing substrate and the increasing adsorbate signal. Both curves show well-defined break points at an evaporation time of $4 \mathrm{~min}$. The strong change in the slope at the break point and the linearity before and after this point indicate that $\mathrm{Ga}$ is growing in a Stranski-Krastanov mode on $\mathrm{Si}(113)$ at 300 $\mathrm{K}$, which means that clustering occurs on top of the first ML. A further proof of this growth mode is provided by the substrate signal at 4 min evaporation time. Assuming an attenuation length of $5.5 \AA$ for the outgoing Si electrons $^{36}$ and a thickness of $2.82 \AA$ for the Ga layer, the $\mathrm{Si}$ Auger signal should be lowered to $60 \%$. This agrees perfectly with our Auger data. According to these results

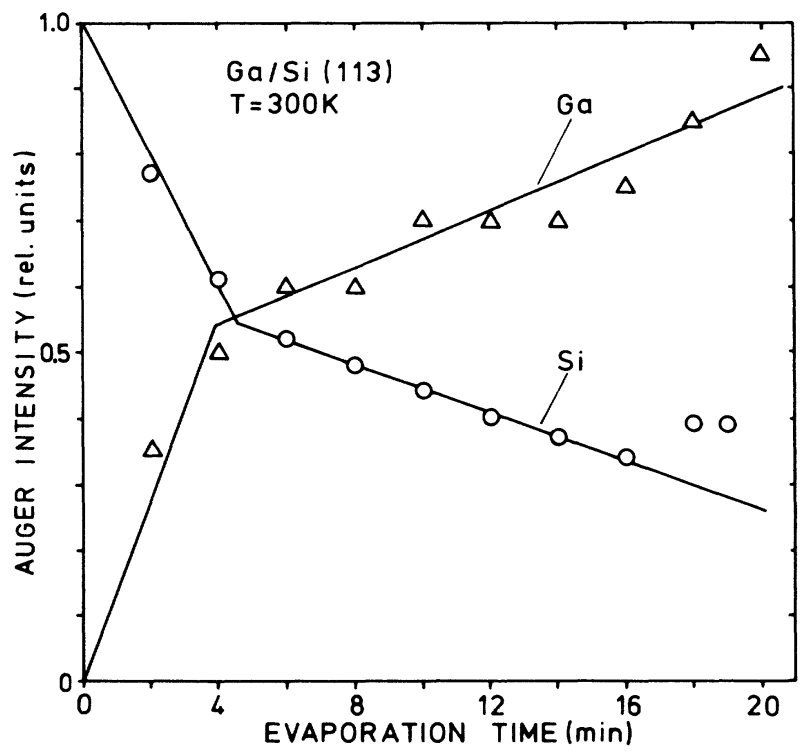

FIG. 1. Ga (53 eV, peak-to-peak, triangles) and $\mathrm{Si}(92 \mathrm{eV}$, negative peak height, circles) Auger intensities depending on the $\mathrm{Ga}$ evaporation time. One monolayer of $\mathrm{Ga}$ corresponds to an evaporation time of $4 \mathrm{~min}$. the monolayer of $\mathrm{Ga}$ corresponds to an evaporation time of $4 \mathrm{~min}$. The growth mode compares well with measurements of Bolmont et al. ${ }^{4}$ on cleaved $\operatorname{Si}(111)(2 \times 1)$ where a similar behavior was found. A slightly different growth mode was documented by Bourguignon et al. for the $\mathrm{Si}(100)$ surface. ${ }^{14}$ On this surface Ga grows in the Frank van der Merwe mode at room temperature whereas at higher temperatures a Stranski-Krastanov growth mode occurs.

Valence-band spectra of the clean and Ga-covered $\mathrm{Si}(113)$ surfaces are presented in Fig. 2. These spectra were obtained from adlayers which were prepared by successive evaporation of $\mathrm{Ga}$. The valence-band spectrum of clean $\mathrm{Si}(113)$ is discussed in Ref. 29. The strong surface state emission near the valence-band edge at $0.9 \mathrm{eV}$ below $E_{F}$ is completely quenched when the $\mathrm{Ga}$ coverage exceeds a third of a monolayer. When the coverage reaches a monolayer a new broad feature develops between 1.5 and $2 \mathrm{eV}$ below $E_{F}$. The surface state at $0.9 \mathrm{eV}$ has its origin in the dangling bonds of the $\mathrm{Si}(113)$ surface. ${ }^{29}$ The decrease of its intensity with $\mathrm{Ga}$ coverage is due to an interaction of the $\mathrm{Ga}$ atoms with the dangling bonds. For $\mathrm{Si}(111)$ the ordered $(\sqrt{3} \times \sqrt{3})$ submonolayer structures of $\mathrm{Al}, \mathrm{Ga}$, and $\mathrm{In}$ have been studied in detail. $^{20-28}$ For these ordered adlayers distinct interface states have been observed by ARUPS and $k$-resolved inverse photoelectron spectroscopy. In the case studied in this work the absence of sharp, $k$-dependent metalinduced features in the submonolayer region is expected, considering the fact that the adlayer is not ordered, as shown by LEED. For a coverage of 2 ML the feature

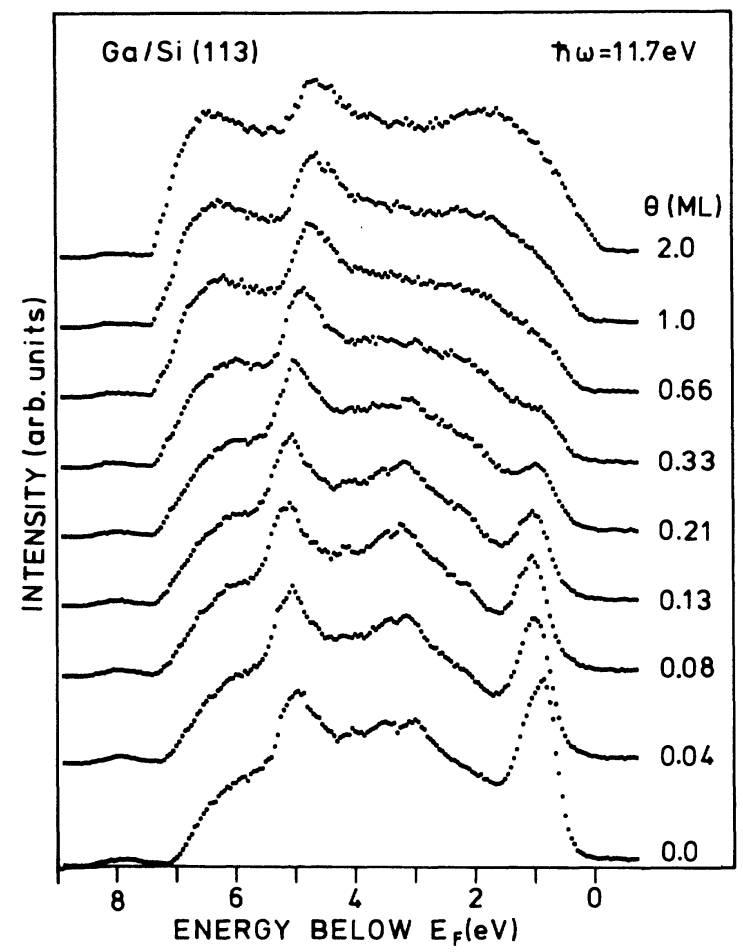

FIG. 2. Angle-resolved valence-band photoemission spectra from $\mathrm{Si}(113)$ as a function of $\mathrm{Ga}$ coverage (normal emission, photon energy $11.7 \mathrm{eV}$ ). The energy is referenced to $E_{F}$; no correction of band bending has been made. 
around $2 \mathrm{eV}$ could be a mixture of broadened interface states and $\mathrm{Ga}$ metal states. The peak at $4.9 \mathrm{eV}$, which is attributed to a bulk transition, ${ }^{29}$ exhibits a shift to higher binding energies after the first two evaporation steps. Upon further evaporation this shift is reversed, and at 2 ML coverage the position of the $4.9-\mathrm{eV}$ peak is shifted to lower binding energies compared with the clean surface. This behavior reflects the band bending which will be discussed in connection with the $\mathrm{Si} 2 p$ core-level shifts.

In order to determine the band bending, $\mathrm{Si} 2 p$ corelevel spectra were taken with a photon energy of $108 \mathrm{eV}$. It has been already demonstrated by several groups ${ }^{2,9,21}$ that at this photon energy photoelectrons are collected from a mean depth of $20-50 \AA .{ }^{36}$ Under these conditions one probes the bulk state, and no surface core-level shifts or chemical shifts of the silicon atoms at the interface influence the band-bending determination. We have completely reproduced the data from the literature. ${ }^{2,9,21}$ The Si $2 p$ spectrum, measured for the clean surface with good energy resolution, is shown in our foregoing contribution ${ }^{29}$ and therefore not presented here again. The escape depth of the outgoing electrons is much smaller than the width of the space-charge region (about $500 \AA$ in our case), so that always the band bending at the surface is measured.

Si $2 p$ core-level spectra for different $\mathrm{Ga}$ coverages are shown in Fig. 3. This set of spectra has been measured within one run of the experiment. The thickness of the $\mathrm{Ga}$ layer was increased without removing the $\mathrm{Ga}$ after

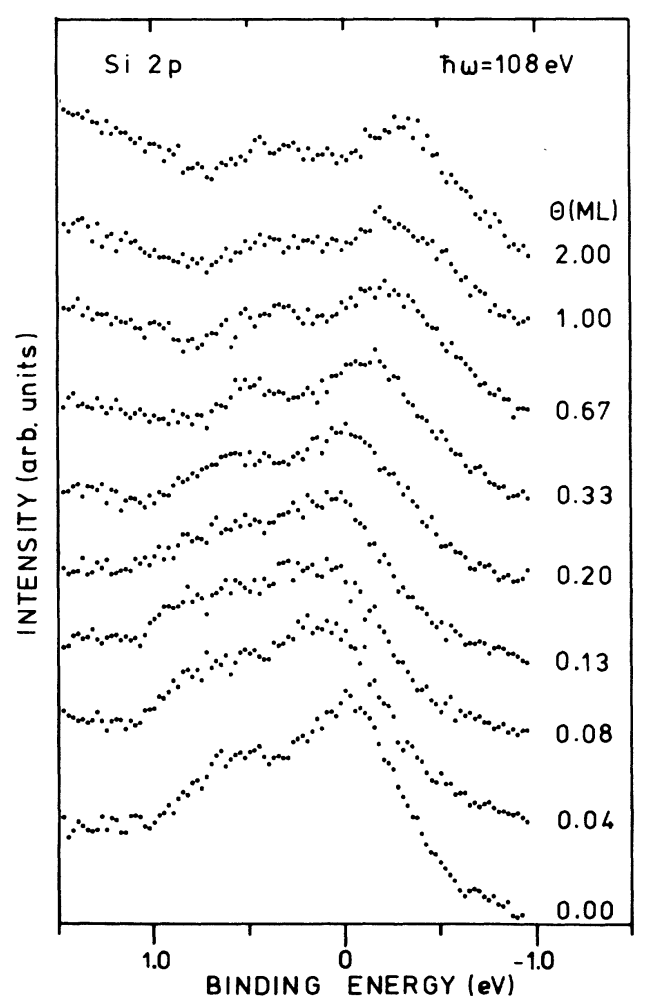

FIG. 3. Photoemission spectra of the Si $2 p$ core level as a function of $\mathrm{Ga}$ coverage on $\mathrm{Si}(113)$ (normal emission; photon energy, $108 \mathrm{eV}$ ). The energy is referenced to the $\mathrm{Si} 2 p_{3 / 2}$ peak of clean $\mathrm{Si}$; no correction of band bending has been made. each measurement. We have decreased the energy resolution and tolerated a signal-to-noise ratio as shown in Fig. 3 in order to keep the measuring time short since the $\mathrm{Ga-covered} \mathrm{surface}$ reacts strongly with residual gases.

The analysis of peak positions from spectra as shown in Fig. 3 gives the relative change of band bending. Together with the Fermi level position of the clean $\mathrm{Si}(113)$ surface the dependence of the Fermi level position from the Ga coverage can be calculated as shown in Fig. 4 (bottom). The Fermi-level position of the clean $\mathrm{Si}(113)$ surface is $0.55 \mathrm{eV}$ above the valence-band maximum, as deduced from photovoltage measurements at $20 \mathrm{~K}$ on the same sample. ${ }^{29}$ Starting from this midgap position, the SB - which is the difference between the Fermi-level position and the valence-band maximum, since our sample is $p$-doped-increases until it reaches its maximum of $0.73 \mathrm{eV}$ at a very low coverage of $0.08 \mathrm{ML} \mathrm{Ga}$. This behavior is called overshooting in the following. From 0.08 ML to higher coverages, the band bending decreases monotonically until the SB height reaches a value of 0.28 $\mathrm{eV}$ for a thickness of $2 \mathrm{ML} \mathrm{Ga}$. This value has to be corrected by $\Delta V_{s}$ for the finite sampling depth $\lambda$ of 20-50 $\AA$ for the photoelectrons. Assuming a linear change of band bending $V_{s}$ with distance and near to the interface, $\Delta V_{s}$ is given by $\Delta V_{s}=\lambda\left(2 n e V_{s} / \epsilon \epsilon_{0}\right)^{1 / 2}$, where $n$ is the density of charge carriers and $\epsilon=11.9$ for Si. This correction amounts to $20-60 \mathrm{meV}$, from which we take $40 \mathrm{meV}$ as a mean value. Therefore, we deduce a SB height of $V_{s}=0.32 \pm 0.1 \mathrm{eV}$. The accuracy is estimated
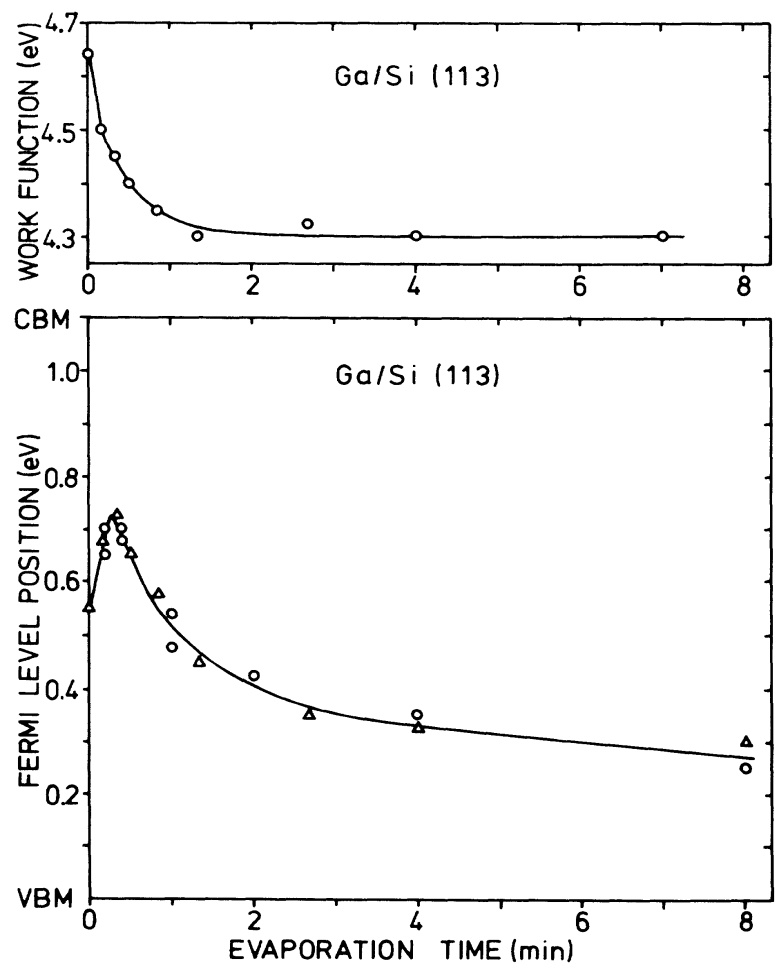

FIG. 4. Work function (top) and Fermi level position in the $\mathrm{Si}$ band gap (bottom) vs the $\mathrm{Ga}$ evaporation time $(4 \mathrm{~min}=1$ $\mathrm{ML}$ ). The band bending was evaluated from the binding-energy shift of the Si $2 p_{3 / 2}$ core level (photon energy, $108 \mathrm{eV}$; two independent measurements). 


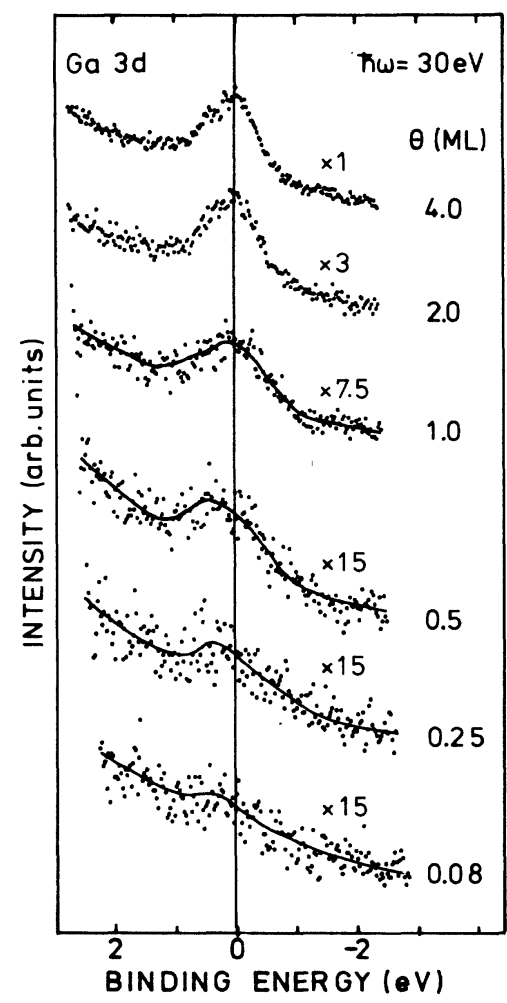

FIG. 5. Photoemission spectra of the $\mathrm{Ga} 3 d$ core level as a function of $\mathrm{Ga}$ coverage on $\mathrm{Si}(113)$ (normal emission; photon energy, $30 \mathrm{eV}$ ). The energy is referenced to the $\mathrm{Ga} 3 d_{5 / 2}$ peak of the metallic adlayer $(4 \mathrm{ML})$. The spectra have been shifted to correct for the band bending.

taking into account all statistical and systematic errors including that of $\lambda$.

The work function of the sample lowers to the $\mathrm{Ga}$ value of $4.3 \mathrm{eV}$ almost within the first third of a monolayer, which can be seen in the upper part of Fig. 4. It is known that two different mechanisms are responsible for work-function changes of a semiconductor surface. The change of the work function can be due to a dipole directly at the surface, which means a charge separation over the length of a few angstroms, or a change in band bending which is due to a charge separation over the width of the space charge layer, typically some hundred angstroms. Any change in dipole at the surface changes the electron affinity and ionization potential $\xi$, i.e., the change in work function $\Delta \phi$ is given by $\Delta \phi=e \Delta V_{s}+\Delta \xi$ where $\Delta V_{s}$ is the change in band bending which is equal to the position of $E_{F}$ in the gap, as shown in the lower part of Fig. 4, minus the value of $E_{F}$ in the bulk. Thus $\Delta \xi$ can /easily be calculated from the results of Fig. 4 . It turned out that upward bending of the bands for coverages greater than $0.08 \mathrm{ML}$ is compensated for by a decrease of $\xi$ so that $\phi$ stays constant beyond $0.3 \mathrm{ML}$. Finally, it should be noted that the work function at zero coverage does not reach the value of $\phi=4.81 \mathrm{eV}$ given in our recent paper. ${ }^{29}$ This is attributed to some lack of ultimate cleanliness when working at the storage ring with synchrotron radiation.
The $\mathrm{Ga} 3 d$ core-level spectra are given as a function of coverage in Fig. 5. At coverages below $1 \mathrm{ML}$ the binding energy is shifted to higher values by about $500 \mathrm{meV}$, referenced to the value of a thick layer. Since band bending is made by a positive charge of about $\frac{1}{600} \mathrm{ML}$ (using simple electrostatics, $\epsilon_{\mathrm{Si}}=11.9,1 \mathrm{ML}=8.2 \times 10^{14} \mathrm{~cm}^{-2}$, $n=2.5 \times 10^{17} \mathrm{~cm}^{-3}$ ) the shift cannot be explained by charging of a measurable quantity of $\mathrm{Ga}$ atoms. Instead, we assume that these $\mathrm{Ga}$ atoms are not in a bulklike state so that any photoelectron hole is not as completely shielded as in the bulk state, giving rise to an increased electronic binding energy. A similar effect was observed for the system $\mathrm{Al}$ on $\mathrm{GaAs}(110)$ (Ref. 37) at very low $\mathrm{Al}$ coverages.

\section{DISCUSSION}

Recently, Klepeis and Harrison ${ }^{30}$ calculated the electronic structure at the metal-Si(100) interface for small amounts of column-III metals. They considered three different coverages: a single adatom, $0.5 \mathrm{ML}$ of metal, and a full ML. For a single neutral adatom the energy $(0,+)$ for the removal of an electron and the energy $(0,-)$ for adding an electron can be calculated. If the $(0,+)$ level lies in the semiconductor band gap but above the Fermi level of the semiconductor, electrons are transferred from the metal atom to the semiconductor, resulting in pinning of the Fermi level at or near the $(0,+)$ level. In the case of aluminum on $\mathrm{Si}(100)$ the $(0,+)$ level has been calculated to be $0.76 \mathrm{eV}$ above the valence-band maximum (VBM). The $(0,-)$ level lies several $\mathrm{eV}$ above the $(0,+)$ level. With increasing coverage the gap between both levels decreases and the $(0,+)$ level moves towards the VBM. In the case of a metallic overlayer both levels are at the position of the metallic Fermi energy.

The behavior we observed is shown in the lower part of Fig. 4. It is very similar to the prediction discussed above. At very low coverages the Fermi level moves up to $0.73 \mathrm{eV}$ above the VBM. This is possible because for our $p$-doped sample the Fermi level lies below the $(0,+)$ level in the case of low coverages. With increasing coverage this process is stopped and a second process takes over moving $E_{F}$ down again until it reaches its final value at a thickness of $2 \mathrm{ML}$. At this coverage the electronic state of the Ga layer is metallic. This is in analogy to the metallization of thin alkali-metal layers. The final Fermi-level position is determined by the metal-induced gap states (MIGS). ${ }^{38-41}$ There are several experimental facts which prove the metallization of the Ga overlayer when a coverage of $2 \mathrm{ML}$ is reached. The work function is $4.3 \mathrm{eV}$ (Fig. 4), which is the known value for Ga metal films. The peak belonging to the $\mathrm{Ga} 3 d$ level assumes its final energy and peak shape at this coverage (Fig. 5). Finally, the most important observation is the emission at $E_{F}$ evolving at this coverage as can be seen from Fig. 2 . Thus the metallization of the adlayer at about $2 \mathrm{ML}$ is proven experimentally, giving support for the occurence of MIGS. Therefore, our assumption, that MIGS determine the final position of $E_{F}$, is very consistent with our experimental observations. The final position of $E_{F}$ de- 
pends on the bulk properties of $\mathrm{Si}$ and $\mathrm{Ga}$ and seems to be rather independent from the special interface or the doping of the Si sample. The latter conclusion is drawn comparing the SB heights found for different interfaces, as discussed below.

Changes of band bending, which are similar to our results, have been observed on $p$-type GaAs(110) for In, ${ }^{31}$ $\mathrm{Al}$, In, and Ag (Ref. 32) and recently for the alkali metals. ${ }^{3,34}$ These interfaces showed overshooting of band bending only at low temperature with the exception of the alkali-metal interfaces. All authors recognized that two different processes are operating here: one at low coverage and another one at higher coverages. Cao et al. ${ }^{32}$ pointed to the fact that, at the low temperature, defect formation is negligible, and found a Schottky-like behavior at low coverages for the $p$-type samples, i.e., a faster movement of $E_{F}$ for a metal with smaller work function. On the other hand, the $n$-type samples did not show Schottky-like behavior, i.e., the position of $E_{F}$ in the gap moved slowly with coverage independent of the metal work function even for a high-work-function metal like $\mathrm{Au}$. Stiles et al. ${ }^{31}$ came to very similar conclusions as Cao et al. ${ }^{32}$ pointing explicitly to the problem with the $n$-type samples. Therefore, the explanation of the low-coverage process by the Klepeis-Harrison model ${ }^{30}$ seems more appropriate since it is consistent for both $n$ and $p$-type samples. In this model the faster movement of $E_{F}$ with coverage for the metal with smaller work function can be explained by the reasonable assumption that the $(0,+)$ level of the metal adatoms moves up in the gap with decreasing work function of the bulk metal. No charge transfer is expected for $n$-type samples and therefore the first process is not working there. Mönch ${ }^{35}$ ex- $^{-}$ plained the findings of Stiles et al. ${ }^{31}$ similarly to the model of Klepeis and Harrison, ${ }^{30}$ calling the first pinning species "adatom-related surface states of donor character." Prietsch et al. ${ }^{33}$ arrived, basically, at the same conclusion for alkali-metal layers on $\mathrm{GaAs}(110)$. In a systematic study they demonstrated that the first adatoms are ionic in character and that the new pinning process occurs only for nonreactive interfaces. Their view was confirmed by Cao et al. ${ }^{34}$

The value of $0.32 \pm 0.10 \mathrm{eV}$, which we found for the SB of $\mathrm{Ga}$ on $\mathrm{Si}(113)$, is in good agreement with measurements for $\mathrm{Ga}$ on $\mathrm{Si}(111)(7 \times 7)$ by Margaritondo et al ., ${ }^{6,7}$ who found $0.85 \pm 0.1 \mathrm{eV}$ for an $n$-type sample which is equivalent to a SB of $0.27 \mathrm{eV}$ for a $p$-type sample. From the MIGS model a value of $0.36 \mathrm{eV}(=1.12-0.76 \mathrm{eV})$ was predicted by Tersoff ${ }^{41}$ for $\mathrm{Si}(110)$, which is in very good agreement with our experimental result. It is interesting to note that the $\mathrm{Si}(113)$ surface offers an independent example to prove the SB to be a bulk property, i.e., independent from the specific crystallographic orientation of the interface. It should be noted also that this agreement is surprisingly good, considering that the values found for the $\mathrm{Si}(111)$ surface by different groups differ largely: $0.15 \mathrm{eV},{ }^{21} 0.27 \mathrm{eV}$ from $0.85 \pm 0.1 \mathrm{eV}$ for $n$-type $\mathrm{Si}(111)(7 \times 7),{ }^{6,7} 0.40 \mathrm{eV}$ from $0.72 \mathrm{eV}$ for $n$-type $\mathrm{Si}(111)(2 \times 1)$ with a good cleave, ${ }^{10} 0.45 \mathrm{eV}$ from $p$-type $\mathrm{Si}(111)(2 \times 1)$ with a good cleave, ${ }^{10} 0.07 \mathrm{eV}$ from $\mathrm{Si}(111)(2 \times 1),{ }^{9}$ and $0.35 \mathrm{eV}$ from $0.77 \mathrm{eV}$ for $n$-type
$\mathrm{Si}(111)(2 \times 1)$. Interestingly, a bad cleave shifted the value from 0.45 to $0.52 \mathrm{eV} .^{10}$

The initial increase or overshooting in band bending has not been found on $\operatorname{Si}(111)(7 \times 7)$ (Refs. 6 and 7) or Si $(111)(2 \times 1)$ (Refs. 9, 13, and 14) surfaces. This could be due to different reasons. First, some earlier experiments on SB's did not examine very low metal coverages. ${ }^{7,9}$ Then, some experiments were carried out with $n$-type samples. $^{3,4}$ A third possible reason may be suggested considering the work of Katnani et al., ${ }^{11}$ who found differences in the development of the SB for In on $\mathrm{Si}(111)(7 \times 7), \mathrm{Si}(111)(2 \times 1)$, and $\mathrm{GaAs}(110)$. The SB on the $(7 \times 7)$ surface reached its final value at much higher coverages than the SB on the $(2 \times 1)$ surface. The SB on GaAs showed a behavior more similar to the development on the $\mathrm{Si}(111)(2 \times 1)$ surface. The authors, therefore, assumed that the density of intrinsic surface states in the band gap is smaller on the $\operatorname{Si}(111)(2 \times 1)$ surface than it is on the $(7 \times 7)$ surface. Since in our case the development of the $\mathrm{SB}$ is as fast as in the case of $\mathrm{Si}(111)(2 \times 1)$, and furthermore our results are similar to results on GaAs(110), we conclude that the density of intrinsic surface states in the band gap is small for $\mathrm{Si}(113)$. Actually, the density of surface states is peaked at $0.9 \mathrm{eV}$ below $E_{F}$ and the tail reaching up to the Fermi edge is only weak in the energy gap, as can be seen from Fig. 2 for the clean surface.

So far, we have stressed the similarity between the $\mathrm{GaAs}(110)$ result and our Si(113) result. Quite obviously, there is also a large difference between these substrates which lies in the fact that there are no surface states and no pinning of $E_{F}$ in the gap for GaAs(110). This explains why the change in band bending is so steep at the beginning. At the $\mathrm{Si}(113)$ surface we start from an already pinned position of $E_{F}$. This pinning counteracts the change introduced by the arriving $\mathrm{Ga}$ atoms. As a result, the change of band bending is not nearly so steep compared to GaAs(110), as can be clearly seen from Fig. 4.

Finally, it should be stressed that, whereas the two different mechanisms in the pinning process seem to be qualitatively understood, a quantitative calculation for a microscopic model is still missing. This model has to work out the transition from the partly ionic to the metallic bonding for an adatom such as $\mathrm{Ga}$ on $\mathrm{Si}$. In part, the absence of such a calculation may be due to the fact that so far no microscopic picture of a simple abrupt and nonreactive SB has been worked out experimentally.

\section{CONCLUSIONS}

During the initial stages of SB formation on $p$-type $\mathrm{Si}(113)$ an overshooting of the band bending is found, similar to results on GaAs(110) surfaces. As far as we know, this overshooting is seen here on a covalent semiconductor for the first time. Its observation supports the calculations of Klepeis and Harrison ${ }^{30}$ in which a most ideal interface is assumed. It seems that overshooting of band bending is an effect common to ideal interfaces with a low density of surface states in the band gap. From the models and explanations given so far it seems that the pinning of $E_{F}$ at low coverages is due to adatom-related surface states of donor character. ${ }^{35}$ The final value of the 
SB height is $0.32 \pm 0.10 \mathrm{eV}$. It is not influenced through the first pinning process and is found to agree with measurements on other silicon surfaces. The final pinning position is consistently interpreted in terms of metalinduced gap states. The $\mathrm{Si}(113)$ surface seems to have interesting properties which are different from other silicon surfaces and which will be further investigated.

\section{ACKNOWLEDGMENTS}

We thank W. Ranke for several discussions and P. Geng for constructing the sample holder and for technical assistance. This work was supported by the Deutsche Forschungsgemeinschaft (SFB6).
${ }^{1}$ F. Flores and C. Tejedor, J. Phys. C 20, 145 (1987); I. Lindau and T. Kendelewicz, CRC Crit. Rev. Solid State Mater. Sci. 13, 27 (1986); L. J. Brillson, Surf. Sci. Rep. 2, 123 (1982).

${ }^{2}$ L. J. Brillson, M. L. Slade, A. D. Katnani, M. Kelly, and G. Margaritondo, Appl. Phys. Lett. 44, 110 (1984).

${ }^{3}$ D. Bolmont, P. Chen, and C. A. Sébenne, J. Phys. (Paris) Colloq. 45, C5-419 (1984).

${ }^{4}$ D. Bolmont, P. Chen, C. A. Sébenne, and F. Proix, Surf. Sci. 137, 280 (1984).

${ }^{5}$ J. J. Lander and J. Morrison, Surf. Sci. 2, 553 (1964).

${ }^{6}$ G. Margaritondo, S. B. Christman, and J. E. Rowe, J. Vac. Sci. Technol. 13, 329 (1976).

${ }^{7}$ G. Margaritondo, J. E. Rowe, and S. B. Christman, Phys. Rev. 14, 5396 (1976).

${ }^{8}$ J. E. Rowe, S. B. Christman, and G. Margaritondo, Phys. Rev. Lett. 35, 1471 (1975).

${ }^{9}$ J. L. Freeouf, M. Aono, F. J. Himpsel, and D. E. Eastman, J. Vac. Sci. Technol. 19, 681 (1981).

${ }^{10}$ J. D. van Otterloo, Surf. Sci. 104, L205 (1981).

${ }^{11}$ A. D. Katnani, N. G. Stoffel, H. S. Edelman, and G. Margaritondo, J. Vac. Sci. Technol. 19, 290 (1981).

${ }^{12}$ G. Platero, J. A. Vergés, and F. Flores, Surf. Sci. 168, 100 (1986).

${ }^{13}$ B. Bourguignon, R. V. Smilgys, and S. R. Leone, Surf. Sci. 204, 473 (1988).

${ }^{14}$ B. Bourguignon, K. L. Carleton, and S. R. Leone, Surf. Sci. 204, 455 (1988).

${ }^{15}$ A. Kawazu, I. Yokohama, H. Suzuki, A. Ohsaki, K. Takeuchi, and H. Sakama, Phys. Rev. B 36, 9809 (1987).

${ }^{16}$ M. Otsuka and T. Ichikawa, Jpn. J. Appl. Phys. 24, 1103 (1985).

${ }^{17}$ T. Thundat, S. M. Mohapatra, B. N. Dev, W. M. Gibson, and T. P. Das, J. Vac. Sci. Technol. A 6, 681 (1988).

${ }^{18} \mathrm{~J}$. Zegenhagen, M. S. Hybertsen, P. E. Freeland, and J. R. Patel, Phys. Rev. B 38, 7885 (1988).

${ }^{19}$ J. Nogami, S. Park, and C. F. Quate, Surf. Sci. 203, L631 (1988); Appl. Phys. Lett. 53, 2086 (1988); J. Vac. Sci. Technol. B 6, 1479 (1988).

${ }^{20}$ G. V. Hansson, R. Z. Bachrach, R. S. Bauer, and P. Chiara- dia, Phys. Rev. Lett. 46, 1033 (1981).

${ }^{21}$ F. J. Himpsel, G. Hollinger, and R. A. Pollak, Phys. Rev. B 28, 7014 (1983).

22J. M. Nicholls, P. Mårtensson, G. V. Hansson, and J. E. Northrup, Phys. Rev. B 32, 1333 (1985).

${ }^{23}$ R. I. G. Uhrberg, G. V. Hansson, J. M. Nicholls, P. E. S. Persson, and S. A. Flodström, Phys. Rev. B 31, 3805 (1985).

${ }^{24}$ G. V. Hansson, J. M. Nicholls, P. Mårtensson, and R. I. G. Uhrberg, Surf. Sci. 168, 105 (1986).

${ }^{25}$ T. Kinoshita, S. Kono, and T. Sagawa, Solid State Commun. 56, 681 (1985).

${ }^{26}$ T. Kinoshita, S. Kono, and T. Sagawa, Phys. Rev. B 32, 2714 (1985).

${ }^{27}$ A. Taleb-Ibrahimi and C. A. Sébenne, Surf. Sci. 168, 114 (1986).

${ }^{28}$ J. M. Nicholls, B. Reihl, and J. E. Northrup, Phys. Rev. B 35, 4137 (1987).

${ }^{29}$ U. Myler and K. Jacobi, Surf. Sci. 220, 353 (1989).

${ }^{30}$ J. E. Klepeis and W. A. Harrison, J. Vac. Sci. Technol. B 6, 1315 (1988).

${ }^{31}$ K. Stiles, A. Kahn, D. G. Kilday, and G. Margaritondo, J. Vac. Sci. Technol. B 5, 987 (1987).

${ }^{32}$ R. Cao, K. Miyano, T. Kendelewicz, K. K. Chin, I. Lindau, and W. E. Spicer, J. Vac. Sci. Technol. B 5, 998 (1987).

${ }^{33}$ M. Prietsch, C. Laubschat, M. Domke, and G. Kaindl, Europhys. Lett. 6, 451 (1988); M. Prietsch, M. Domke, C. Laubschat, T. Mandel, C. Xue, and G. Kaindl, Z. Phys. B 74, 21 (1989).

${ }^{34}$ R. Cao, K. Miyano, T. Kendelewicz, I. Lindau, and W. E. Spicer, Appl. Phys. Lett. 54, 1250 (1989).

${ }^{35}$ W. Mönch, J. Vac. Sci. Technol. B 6, 1270 (1988).

${ }^{36}$ M. P. Seah and W. A. Dench, Surf. Interf. Anal. 1, 1 (1979).

${ }^{37}$ R. R. Daniels, A. D. Katnani, T. X. Zhao, and G. Margaritondo, Phys. Rev. Lett. 49, 895 (1982).

${ }^{38}$ V. Heine, Phys. Rev. 138, A 1689 (1965).

${ }^{39}$ E. Louis, F. Yndurain, and F. Flores, Phys. Rev. B 13, 4408 (1976).

${ }^{40}$ M. L. Cohen, Adv. Electron. Electron Phys. 51, 1 (1980).

${ }^{41}$ J. Tersoff, Phys. Rev. Lett. 52, 465 (1984). 